\title{
Evaluation of the neuroprotective effects of anti-inflammatory drugs in experimental spinal cord damage
}

\author{
Durna $\mathrm{F}^{1}$, Aykanat $\mathrm{O}^{1}$, Gurses $\mathrm{L}^{2}$, Bavbek $\mathrm{M}^{3}$ \\ Department Neurosurgery, Ahi Evran University Training \& Research Hospital, Kirsehir, Turkey. \\ dromeraykanat@gmail.com
}

\begin{abstract}
In this study, we aimed to investigate the effectiveness of etofenamate and compare it with methyl prednisolone in the experimental spinal cord trauma model. A total of 31 Wistar Albino rats weighed between 220 and $270 \mathrm{gr}$ were used in this study. The rats were divided into three groups as the control; posttraumatic normal saline (NS), trauma + E; posttraumatic etofenamate; and trauma + methylprednisolone, posttraumatic methylprednisolone. All medications were given into the peritoneum. Six hours after trauma and drug administration, approximately $2 \mathrm{~cm}$ of cord segment in the area subjected to dorsal laminectomy was dissected from the dura spinal cord and removed. The samples were histopathologically examined. In this study, significant differences were found both between trauma + NS and trauma + methylprednisolone, and between trauma NS and trauma + etofenamate, and trauma + methylprednisolone and trauma + etofenamate groups according to the Ivan Damjanov criteria and in terms of petechial hemorrhage, diffuse bleeding, loss in the regulation of grey and white matters, edema, necrosis, and cystic degeneration findings. According to the Ivan Damjanov criteria, trauma + NS group was found as Grades 2-3, trauma + etofenamate group as Grade 1, and trauma + methylprednisolone as Grades 1-2. Neuroprotective effect of etofenamate was found to be stronger than that of methyl prednisolone in rats with induced posttraumatic spinal cord damage (Tab. 4, Ref. 24). Text in PDF www.elis.sk.

KEY WORDS: etofenamate, methylprednisolone, spinal trauma, rats.
\end{abstract}

\section{Introduction}

Today, spinal cord damage (SCI) presents as a process, which is untreatable, but can be rehabilitated in all organisms. The incidence of acute spinal cord injuries is increasing year by year. Considering lifelong treatment and care costs, workforce and income losses, and social and psychological problems they face; patients exposed to spinal cord injury present a serious health problem affecting patients, their families, and country economy. Despite the advancements in spinal biomechanics and surgical techniques, and the developments in rehabilitation process, no significant improvements were achieved for the neurologic healing. Although numerous studies and researches were conducted on the injury of medulla spinalis, a medical option to repair the damage is yet to be developed $(1,2)$.

Spinal cord trauma (SCT) causes the series of events leading to secondary neuronal cellular damage. Although the main mechanism leading to SCT is not fully understood, early inflammatory response in the cord subjected to acute damage is thought

${ }^{1}$ Department Neurosurgery, Ahi Evran University Training \& Research Hospital, Kirsehir, Turkey, ${ }^{2}$ Department Neurosurgery, Health Sciences University, Diskapi Yildirim Beyazit Training \& Research Hospital, Ankara, Turkey, and ${ }^{3}$ Department Neurosurgery, Yildirim Beyazit University, Medical School, Ankara, Turkey

Address for correspondence: O. Aykanat, Department Neurosurgery, Ahi Evran University Training \& Research Hospital, Kirsehir, Turkey Phone: +90.386.2134515, Fax: +90.386.2134519 to trigger the secondary tissue damage. Inflammatory damage begins with the filtration of neutrophils causing the activation of microglia, within hours following a trauma. This is followed by the secondary response, which attempts to localize the damage. Since prevention of the primary damage by trauma is not possible, one can thought to prevent the inflammatory response, which plays a role in the pathogenesis of the secondary damage (3). Therefore, cell replacement and therapeutic genetic transfers are being tested (4). However, a practical treatment method to prevent early inflammatory response has not yet been developed. Prevention of inflammatory cellular infiltration after spinal cord injury has been shown to be effective in the protection of damaged spinal cord and long- term improvement of sensory, motor and autonomic functions (5). The important thing here is to provide the balance between the destructive type infiltrative pro-inflammatory neutrophils and macrophages, and subsequent beneficial effects of macrophages and T-cells.

Etofenamate exerts its antiinflammatory effect via the inhibition of prostaglandin synthesis (6). It prevents prostaglandin synthesis through the inhibition of cyclooxygenase enzyme.

Whereas methylprednisolone shows its antiinflammatory effect by inhibiting phospholipase A2 enzyme, and thus reduces not only the synthesis of cyclooxygenase products, but also the synthesis of lipoxygenase (6).

The objective of this study was to investigate effects of etofenamate, which is an antiinflammatory agent that would halt early, destructive, and leukocyte mediated actions and enable wound 
207-211

healing in long term regenerative process in spinal cord injury, on neurological damage and histological changes in the spinal tissue sections in a rat spinal cord trauma model, and to compare the results with the effects of methylprednisolone, which is among the strongest known anti-inflammatory glucocorticoid agents.

\section{Material and methods}

The study material composed of 31 healthy Wistar Albino male rats weighed between 220 and $270 \mathrm{~g}$. The procedures on the rats included in this study were performed in line with the decisions of the ethics committees of the Ministry of Health, Ankara Diskapi Yildirim Beyazit Training and Research Hospital, and Ankara University, Faculty of Veterinary Science. The animals were fed with standard rat pellet feed and tap water, and kept in the cage with 12-hour dark / light cycle. The principles by the USA, National Health Institute were followed during the experiments.

\section{Groups}

The subjects were randomly divided into 3 separate groups:

Group 1 (Control group $(C), n=10$ ): Ten rats in this group were given intraperitoneal normal saline after the induction of spinal cord trauma.

Group 2 (Trauma + Etofenamate group (TE), $\mathrm{n}=11$ ): Eleven rats in this group were administered $20 \mathrm{mg} / \mathrm{Kg}$ intraperitoneal etofenamate after the induction of spinal cord trauma.

Group 3 (Trauma + Methylprednisolone (TMP), $\mathrm{n}=10$ : Ten rats in this group were administered $30 \mathrm{mg} / \mathrm{Kg}$ intraperitoneal methylprednisolone after the induction of spinal cord trauma.

\section{Anesthesia and the induction of trauma}

Rats were deprived of food the night before, and their weights were determined. The rats were then anesthetized by intraperitoneal injection of a mixture of $12 \mathrm{mg} / \mathrm{Kg}$ xylazine $\mathrm{HCI}$ and $75 \mathrm{mg} / \mathrm{Kg}$ ketamine. Maintenance was provided by administration of these medications by half doses when necessary. Rats were allowed to breath spontaneously. After the corneal reflex and anesthesia depth were controlled with tail squeezing method, rats' physiological values such as: breathing, pulse and rectal temperature were recorded at the hours 0 and 6 (Tabs 1 and 2). The rats were given prone position, and the operation site was prepared. Following a $2-3 \mathrm{~cm}$ incision on the skin, the paravertebral muscles were excluded with a blunt dissection, and dorsal laminectomy was applied at the level of T7 - T8. A pin of $0.3 \mathrm{~mm}$ thickness and $10 \mathrm{~g}$

Tab. 1. Distribution of pretraumatic (0th hour) physiological parameters between the groups.

\begin{tabular}{lccc}
\hline & $\begin{array}{c}\text { Control } \\
(\mathrm{n}=10)\end{array}$ & $\begin{array}{c}\text { Trauma+ } \\
\text { Etofenamate } \\
(\mathrm{n}=11)\end{array}$ & $\begin{array}{c}\text { Trauma+ } \\
\text { Methyl prednisolone } \\
(\mathrm{n}=10)\end{array}$ \\
\hline Weight & $301.4 \pm 9.3$ & $305.7 \pm 6.3$ & $308.9 \pm 10.4$ \\
Rectal Temperature & $36.5 \pm 0.3$ & $36.4 \pm 0.3$ & $36.5 \pm 0.3$ \\
Respiratory Rate & $128.1 \pm 0.8$ & $131.3 \pm 0.9$ & $130.4 \pm 1.1$ \\
Heart Rate & $235.5 \pm 5.2$ & $232.4 \pm 5.7$ & $230.2 \pm 4.3$ \\
\hline
\end{tabular}

The values are given as the mean \pm standard deviation
Tab. 2. Distribution of posttraumatic (6th hour) physiological parameters between the groups.

\begin{tabular}{lccc}
\hline & $\begin{array}{c}\text { Control } \\
(\mathrm{n}=10)\end{array}$ & $\begin{array}{c}\text { Trauma+ } \\
\text { Etofenamate } \\
(\mathrm{n}=11)\end{array}$ & $\begin{array}{c}\text { Trauma+ } \\
\text { Methyl prednisolone } \\
(\mathrm{n}=10)\end{array}$ \\
\hline Weight & $300.9 \pm 9.7$ & $304.4 \pm 8.8$ & $303.7 \pm 6.4$ \\
Rectal Temperature & $36.9 \pm 0.3$ & $36.7 \pm 0.3$ & $36.1 \pm 0.3$ \\
Respiratory Rate & $133.2 \pm 0.9$ & $134.6 \pm 0.5$ & $136.8 \pm 1.0$ \\
Heart Rate & $242.1 \pm 6.9$ & $240.4 \pm 7.1$ & $239.6 \pm 5.5$ \\
\hline
\end{tabular}

The values are given as mean \pm standard deviation.

weight $(50 \mathrm{~g} / \mathrm{cm}$ ) was left to free vertical fall using a prepared assembly, through a measured pipe from a height of $5 \mathrm{~cm}$ on the spinal cord segment, which was exposed without any damage to the dura mater (approximately 35 minutes after the induction of anesthesia). The incision line was routinely closed post-traumatically. The planned intraperitoneal administration of normal saline, etofenamate and methylprednisolone was performed in the groups. Rectal temperature of the rats was measured during anesthesia and an electrical heater was used to keep the temperature within the range of $35-37^{\circ} \mathrm{C}$. The rats with spontaneous breathing were left to rest and observed to awake without problems. Repeat anesthesia was applied with xylazine and ketamine at the end of the 6th hour. Then, after the necessary site cleaning and covering, all rats underwent thoracotomy incision. Following thoracotomy, the left ventricle was cannulated with a $14 \mathrm{G}$ catheter. The right atrial appendix was opened, and $10 \%$ formalin was given to the left ventricle in $500 \mathrm{~mL}$ NS. Perfusion was continued until clear fluid was observed from the right atrium. Circulation of perfusion fluid was provided by a short time open heart massage after ventricular fibrillation. After clear fluid came from the right atrium and the heart completely stopped, the rats were given prone position. After the cleaning with iodine solution, the closed skin incision was opened, and an approximately $2 \mathrm{~cm}$ cord segment on the area subjected to dorsal laminectomy was dissected from the dura spinal cord and removed. The removed tissue samples were fixed in $10 \%$ formalin solution. The samples were then evaluated under light microscope.

\section{Histopathological evaluation}

The spinal cord segment removed for microscopic examination was fixed in $10 \%$ buffered formalin solution. The tissue samples in formalin fixation were cut into $2-3 \mathrm{~mm}$ sections with proper size, and after being washed with tap water for 5 hours, the samples were dehydrated at alcohol series of $50^{\circ}, 70^{\circ}, 80^{\circ}, 96^{\circ}$ and $100^{\circ}$, and then kept respectively in xylol, paraffin with xylol and paraffin of $56-58{ }^{\circ} \mathrm{C}$ for each two hours and blocked in paraffin. The sections of $4-6$ micron thickness, taken from each paraffin block with microtome, were stained with hematoxylin-eosin (H\&E) and examined under a light microscope. Histopathologic examination was performed by an experienced histopathologist, who was not aware of the treatment and the status of the subjects. Specimen examination of all the groups was separately assessed according to the Ivan Damjanov criteria (Tab. 3) and in terms of petechial hemorrhage, diffuse bleeding, loss in the regulation of 
Tab. 3. Ivan-Damjanov Criteria.

\begin{tabular}{ll}
\hline Grade 0 & Normal: No histological damage finding. \\
\hline Grade 1 & $\begin{array}{l}\text { Mild Damage: Clustering of hemosiderin loaded } \\
\text { macrophages, foamy macrophages, nerve cell loss } \\
\text { with white matter vacuolization, and primarily } \\
\text { affected posterior colons that are the minor findings } \\
\text { of traumatic damage. }\end{array}$ \\
& $\begin{array}{l}\text { Moderate Damage: Loss of grey matter with central } \\
\text { cavitation, foamy macrophages found in the cystic le- } \\
\text { sions. }\end{array}$ \\
\hline Grade 3 & $\begin{array}{l}\text { Severe Damage: Excessive cystic necrosis in the grey } \\
\text { and white matters, presence of gliotic thin peripheral } \\
\text { rim in the residual spinal cord. }\end{array}$ \\
\hline
\end{tabular}

grey and white matters, edema, necrosis, and cystic degeneration findings.

\section{Results}

Petechial bleeding was observed in each case, while almost no diffuse bleeding was observed in the TE group and was significantly decreased in TMP group compared to C Group. An advanced degree of loss in the regulation of grey and white matters was observed in $\mathrm{C}$ group, while this loss was minimal in TE and TMP groups. Edema was marked in C Group, and minimal in TE and TMP groups. Similarly, necrosis was significant in C group, and minimal and isolated in TE and TMP groups. Cystic degeneration was significant in C group and rare in TMP group, while no cystic degeneration was observed in TE group.

According to the Ivan Damjanov Criteria; C Group was found as Grades 2 - 3, TE group as Grade 1, and TMP group as Grades $1-2$.

In conclusion, TE group (Group 2) was found to be significantly protected against edema and hemorrhage.

\section{Histopathological results}

A loss in the regulation of grey and white matters, diffuse hemorrhage and congestion, marked vascular thrombus formation, significant edema and cystic degeneration were observed in the control group (Image 1). Minimal loss in the regulation of grey and white matters, hemorrhage, and focal congestion, minimal edema were observed, while no thrombus formation, and no degeneration were found in the group, which was given etofenamate after trauma (TE) (Images 2 - 3). Whereas mild loss in the regulation of grey and white matters, hemorrhage, diffuse congestion, minimal edema and cystic degeneration were found in the group, which was administered methylprednisolone (TMP) after trauma (Images 4, 5). Evaluation of the pathologies by groups shows Table 4.

\section{Discussion}

Spinal cord damage (SCI), which occurs due to spinal cord trauma, remains an important problem, because its incidence is high in general population, causes advanced physical and psychosocial damage in patients, and an internationally recognized treatment protocol could not be made. Many experimental models of spinal cord trauma have been developed in order to eliminate this crucial problem. Weight fall or "blockingweight" are the most commonly used trauma models $(7,8)$. Weigh fall method was described for the first time by Allen in a dog, and later was adapted to the other animals including rats (9). An acute spinal cord trauma model must create damage similar to spontaneous incident cases, producing consistent sample responses in the spinal cord. For this purpose, we preferred the most commonly used method, which was described by Allen in 1911. Shortly this method based on dorsal laminectomy and falling of a known weight through a tube with of definite length. The created clash is recorded as gram-centimeters. Although laminectomy has been shown to reduce spinal cord blood flow, degree of this fall has been shown not to be as effective as to adversely influence energy metabolism of the spinal cord.

Experimental animals such as: rats, monkeys, dogs and cats are used in experimental spinal cord injury models $(10,11,12)$. Among these, rats are the most preferred animals. Rats are preferred especially in the studies, where body size is not important. In addition, rats are inexpensive. Rat contusion and compression studies have become a standard in SCI experiments. In our study, we used 31 health Wistar Albino male rats, and thus deviation due to race and size was avoided.

The prevention of inflammatory cellular infiltration after spinal cord injury has been shown to be effective in the protection of damaged spinal cord and long- term improvement of sensory, motor and autonomic functions (11). The important thing here is to provide the balance between destructive type infiltrative pro-inflammatory neutrophils and macrophages, and subsequent beneficial effects of macrophages and T-cells. Neutrophils and hematogenously spread macrophages reach to the highest number in the spinal cord at 12th hour, and 5 th -7 th days, releasing pro-inflammatory cytokines, reactive oxygen and NO (nitrogen oxide) $(13,14)$. Cytotoxic agents released by neutrophils and macrophages damage the grey and white matters, leading to scar formation (15).

Cellular death occurring with necrotic and apoptotic mechanism is known to play a role in spinal cord trauma. Microglia cells are thought to release cytotoxic cytokines, leading to the death of oligodendrocytes. In this study, we chose etofenamate, which is strong anti-inflammatory agent, to demonstrate its effect on prevention of inflammation that plays a role in the pathogenesis of secondary damage in the spinal cord, and compared it with methylprednisolone.

Tab. 4. Histopathologic Distribution of the Groups.

\begin{tabular}{lccccc}
\hline & Petechial Hemorrhage & Diffuse Bleeding & Edema & Necrosis & Cystic Degeneration \\
\hline Control Group (Trauma+NS) & + & + & + & & + \\
TE Grubu (Trauma+Etofenamate) & + & - & \pm & + & \pm \\
\hline
\end{tabular}


207-211

Methylprednisolone sodium succinate showed its inflammatory effect by inhibiting phospholipase A2 enzyme. This decreased the synthesis of the products of both cyclooxygenase and lipoxygenase. Although there are numerous clinical and laboratory studies, the role of glucocorticoids in traumatic SCI could be only poorly explained. Glucocorticoids have been used for the first time in acute SCI in 1960s. NASCIS (National Acute Spinal Cord Injury Study) II demonstrated the clinical benefit of high dose intravenous treatment of methyl prednisolone. According to many researchers, high dose of glucocorticoids administered within the first 8 hours after acute SCI is beneficial. However, it should be known that opposite views against NASCIS studies have been reported, and many researchers debate about the use of methylprednisolone in SCI. The first reasonable use of glucocorticoids is based on the hypothesis that glucocorticoids should reduce posttraumatic spinal cord edema as they decrease peritumoral cerebral edema. The first experimental studies were conducted on comparison of placebo, hypothermia, and glucocorticoids in treatment of SCI in dogs. In that study, anti-inflammatory response was changed and neurologic recovery was improved, when hypothermia and steroid treatment were applied immediately after the damage. Although the effect was mild, steroids have been used by clinicians. Experimental studies found that methylprednisolone significantly prevented secondary injuries, was more efficient than the other drugs, and found the widest usage area in routine practice. Methylprednisolone plays an important role in the prevention of posttraumatic spinal cord ischemia, regulation of energy metabolism, arrangement of the extracellular calcium, provision of nerve impulse conduction, and loss of cholesterol from the damaged spinal cord, and inhibition of the release of free fatty acids and eicosanoids (18). However, there are several factors limiting and making difficult the use of high dose MPSS in acute SCI. These factors include side effects due to receptor activity of glucocorticoids (infection, pneumonia, septic shock, diabetic complications, and delayed wound healing), difficulty in calculation and administration of the dose because of sharp biphasic dose response curve, the need for maintaining the application depending on start time of the treatment, possibility of increased damage by treatment applied after 8 hours, glucocorticoids application that may inhibit axonal sprouting, and suppress degenerative responses. (19). Nevertheless, there are some data indicating that steroids inhibit neuron regeneration, and they are thought to make this by inhibiting immune cellular activity.

Etofenamate [2-(2-hydroxyethoxy)ethyl-N-(alpha, alpha, alpha-trifluoro- m-tolyl)-anthranilate] is an analgesic, antipyretic, and non-steroid anti-inflammatory (NSAI) agent. Etofenamate, which is a cyclooxygenase inhibitor, provides its inflammatory effect through the inhibition of prostaglandin synthesis. Its antiinflammatory effect is likely due to inhibition of prostaglandin synthesis, release of bradykinin, histamine and lysosomal enzymes, hyaluronidase and foreign body reactions (6). After parenteral administration of etofenamate; t VT. max was found as approximately 2 hours, t max as 5.4 hours, $\mathrm{C}$ max as $0.633 \mathrm{mmol} / \mathrm{L}$. It binds to proteins by $98-99 \%$. The elimination of etofenamate mainly occurs in the form of free or conjugated hydroxyl flufe- namic acid metabolites as about $35 \%$ with urine and most of the remaining part by the bile. Elimination half-life is approximately 1.9 hours, terminal half-life is 35.4 hours. In conclusion; it has a very early beginning ( $1 / 2-1$ hour) and long lasting (more than 24 hours) effect (6).

Several studies evaluated the histological changes seen after spinal cord ischemia in different ways $(20,21)$. Some authors could demonstrate ischemia specific changes in the spinal cord, whereas the others could not find a change in the spinal cord. Similar to our study, many researchers in literature examined the samples stained with hematoxylin eosin under a light microscope in order to evaluate the changes in the spinal cord following spinal cord ischemia $(20,21)$. In two different studies, Mutch et al. used the ratio of the neuron showing eosinophilic granulation in the anterior spinal cord and accepted dead, to the undamaged neurons in order to reveal histological changes (21). Ivan Damjanov criteria that have been widely used in other studies, were used for pathologic examination $(22,23)$. In our study, the results of the control group were poorer than those of the groups that received methylprednisolone and etofenamate. Good results of the etofenamate groups were remarkable.

Early histopathologic changes following the spinal cord injury included a wide disseminated extravasation of erythrocytes and neutrophils. In our study, less hemorrhage was observed in the etofenamate group compared to the control and the methylprednisolone groups. Histopathological changes in the nerves and supportive glial tissues were seen within 4 hours (24). In the present study, cystic degeneration and necrosis were lower in the etofenamate group than in the other groups.

A disadvantage of our study includes that neurologic examination of the rats could not be performed in a 6-hour study, and reflections of the used agent on neurologic examination could not be provided.

In the light of the data obtained from this study; we demonstrated that significant histological and clinical changes occurred in the spinal cord trauma model in rats, the spinal cord was histologically protected from the secondary effects of trauma in the etofenamate administered group. The subjects in the methyl prednisolone administered group were better protected from the secondary effects of trauma compared to the subjects in the control group, but the protection was weaker than the etofenamate administered group. This study indicates that histologic neuroprotective effect of etofenamate is better than that of methylprednisolone (Tab. 4).

\section{Conclusion}

In conclusion; it was demonstrated with the present study that neuroprotective effect of etofenamate in acute spinal cord damage was stronger compared to methylprednisolone, and it might be a protective agent against traumatic spinal cord damage. We reached a conclusion of that etofenamate might be a useful option in the treatment of spinal cord damage in humans. 


\section{References}

1. Bracken MB, Shepard MJ, Collins WF. Methylprednisolone or naloxone treatment after spinal cord injury. J Neurosurg 1992; 76: 23-31.

2. Geisler FH, Dorsey FC, Coleman WP. Recovery of motor functiona randomized, placebo controlled trial with GM-1 ganglioside. N Eng J Med 1991; 324: 1829-1838.

3. Bartholdi D, Schwab ME. Methylprednisolone inhibits early inflammatory processes but not ischemic cell death after experimental spinal cord lesion in the rat. Brain res 1995; 672: 177-186.

4. Lu J, Ashwell KW, Waite P. Advances in secondary spinal cord injury. Spine 2000; 25 (14): 1859-1866.

5. Taoka Y, Okajima K, Uchiba M, Murakami K, Kushimoto S, Johno M, Naruo M, Okabe H, Takatsuki K. Role of neutrophils in spinal cord injury in the rat. Neuroscience 1997; 79: 1177-1182.

6. Kayaalp SO. Medical Pharmacology for Rational Treatment, Ankara, Feryat Printing Volume 2: 2006-2007, 1990

7. Nystrom B, Berglund JE. Spinal cord restitution following compression injuries in rats. ACTA Neurol Scand. 1998; 78: 467-472.

8. Perez-Espejo MA, Haghighi SS, Adelstein EH, Madsen R. The Effects of taxol methylprednisolone, and 4-aminopyridine in compressive spinal cord injury: A Qualitative Experimental Study. Surg Neurol 1996; 46: 350-357.

9. Farooq M. Spinal Cord Compression Injury in the mouse: presentation of a model including assessment of motor dysfunction. Acta Neuropathol 2000; 100: 13-22.

10. Koyanagi I, Tator HC, Theriault E. Silicone Rubber Microangiography of acute spinal cord injury in the rat. Neurosurg 1993; 32 (2): 260-268.

11. Mabon PJ, Weaver LC, Dekaban GA. Inhibition of monocyte/macrophage migration to a spinal cord injury site by an antibody to theintegrinalphaD: a potential new anti-inflammatory treatment. Exp Neurol 2000; 166: 52-64.

12. Braun J, Bollovv M, Neure L. Use of immunohistological and in situ hybridization techniques in the examination of sacroiliac joint biopsy specimens from patients with ankylosing spondylitis. Arthritis Rheum 1995; 38: 499-505.
13. Cruse JM, Lewis RE, Bishop GR, Kliesch WF, Gaitan E, Britt R. Decreased immune reactivity and neuroendocrine alterations related to chronic stress in spinal cord injury and stroke patients. FASEB J 1994; 8: A113.

14. Turner ML. Cell adhesion molecules: A unifying approach topographic biology. Biol Rev 1992; 67: 359-377.

15. Jones LL, Yamaguchi Y, Stallcup WB, Tuszynski MH. NG2 is a majör chondroitinsulfat proteoglycan produced after spinal cord injury and is expressed by macrophages and oligodendrocyte progenitors. J Neurosci 2002; 22: 2792-2803.

16. Hurlbert RJ. Methylprednisolone For Acute Spinal Cord Injury: an inappropriate standard of care. J Neurosurg 2000; 93 (Suppl): 175-179.

17. Nesathurai S. Steroids Spinal Cord Injury: revisiting the NASCIS 2 and NASCIS 3 trials. J Trauma. 1998; 45: 1088-1093.

18. Perez-Espejo MA, Haghighi SS, Adelstein EH, Madsen R. The Effects of taxol methylprednisolone, and 4-aminopyridine in compressive spinal cord injury: A Qualitative Experimental Study. Surg Neurol 1996; 46: 350-357.

19. Gale K, Kerasidis H, VVrathalI JR. Spinal Cord Contusion in the rat: behavioral analysis of functional neurologic impairment. Experimental Neurology 1982; 88 (1): 123-134.

20. Ducati A, Schieppati M, Giovanelli MA. Effects of deep barbiturate coma on acute spinal cord injury in the cat. Surgical Neurology 1984; 21: 405-131

21. Mutch WAC, Graham MR, Halliday WC. Paraplegia Follovvingthoraci Aortic Cross-clamping in dogs. No difference in neurological outcome with a barbiturate versus isoflurane. Stroke 1993; 24: 1554-60.

22. Tator CH, Fehlings MG. Revievv of the secondary injury theory of acutes pin cord trauma with emphasis on vascular mechanisms. J Neurosurg 1991; 75 (1): 15-26.

23. Tator CH. Pathophysiology And Pathology of spinal cord injury. Neurosurgery 1996; 2847-59.

24. Rawe SE, Lee WA, Perot PL. The Histopathology of experimental spinal cord trauma: The Effect of systemic blood pressure. J Neurosurg. 1978; 48 (6): 1002-1007. 